\title{
B-02
}

\section{EVALUACIÓN CUANTITATIVA Y CUALITATIVA DEL CULTIVO DE AJO MORADO BAJO APLICACIÓN OPTIMIZADA DE VOLÚMENES LIMITADOS DE AGUA DE RIEGO}

\author{
Léllis, B.C. ${ }^{1}$, Pardo, J.J.2 ${ }^{2}$, Tarjuelo J.M. ${ }^{3}$, Martínez-Romero A. ${ }^{4}$ \\ Domínguez $A .^{5}$
}

1 Ingeniero Agrónomo, Investigador, Centro Regional de Estudios del Agua (CREA) UCLM,Ctra. De Las Peñas km. 3,2, 02071 Albacete, brunolellismaria@yahoo.com.br

2 Ingeniero Agrónomo, Investigador, Centro Regional de Estudios del Agua (CREA) UCLM,Ctra. De Las Peñas km. 3,2, 02071 Albacete, josejesus.pardo@uclm.es

${ }^{3}$ Dr. Ingeniero Agrónomo, Catedrático de Universidad; Departamento de Producción Vegetal y Tecnología Agraria, UCLM; jose.tarjuelo@uclm.es

${ }^{4}$ Dr. Ingeniero Agrónomo, Profesor Asociado; EIIAB, Departamento de Mecánica Aplicada e Ingeniería de Proyectos, UCLM; angel.mromero@uclm.es

5 Dr. Ingeniero Agrónomo, Profesor Contratado Doctor; Departamento de Producción Vegetal y Tecnología Agraria, UCLM; Alfonso.dominguez@uclm.es

\section{Resumen}

Castilla-La Mancha (C-LM) es una región de clima semiárido con escasez de recursos hídricos para el regadío. De las variedades de ajo utilizadas en la región, el Ajo Morado de las Pedroñeras presenta valores añadidos, tanto en aspectos económicos, como culturales y de calidad nutricional. Todo ello, unido al elevado coste de la energía asociada a los sistemas de riego presurizados, hace que muchos productores demanden estrategias para mejorar la eficiencia en el uso del agua y hacer más rentable el cultivo. El objetivo principal de este trabajo, es definir y validar estrategias de manejo del riego deficitario controlado, basadas en las metodologías año meteorológico típico (TMY) y del riego deficitario optimizado por etapas (ORDI) que distribuyan de manera óptima un volumen de agua de riego limitado durante las distintas etapas de desarrollo fenológico del Ajo Morado de Las Pedroñeras. Para ello, se ensayaron 5 tratamientos, uno sin déficit y cuatro con distintos volúmenes máximos de agua de riego disponible, correspondientes al 100 (T100), 90 (T90), 80 (T80) y 70\% (T70) de las necesidades netas de riego del cultivo para las condiciones climáticas de un año meteorológico típico (TMY) intermedio en C-LM (fijadas en 3400 $\mathrm{m}^{3} / \mathrm{ha}$ ). Como consecuencia de las condiciones climáticas del año del ensayo, los tratamientos no alcanzaron los niveles de déficit hídrico deseados. No se observaron diferencias significativas en cuanto a rendimiento y calidad para los tratamientos T90, T80 y T70.

\section{1- Introducción}

El ajo (Allium sativum L.) es uno de los cultivos más difundidos en el mundo, con cerca de 1,5 millones de hectáreas plantadas, generando un movimiento de alrededor de 500 millones de dólares por año (FAO, 2016). En Europa existen aproximadamente 108.000 ha del cultivo, de las cuales 21.000 ha pertenecen a España. En la comunidad autónoma de Castilla-La Mancha se localiza la principal zona productora de ajo española, con el 58\% del total nacional (MAGRAMA, 2016). 
De las variedades utilizadas en la zona, el Ajo Morado de las Pedroñeras presenta valores añadidos, tanto en aspectos económicos, como culturales y de calidad nutricional. Sin embargo, la introducción en los últimos años de variedades de ajo blanco, con mayores rendimientos y con menores costes de producción, dificulta la competencia del Ajo Morado en el mercado.

Más del 95\% del área cultivada de ajo en C-LM es bajo regadío (MAGRAMA, 2016) y debido a la escasez de recursos hídricos en la región, los regantes tienen limitada en amplias zonas la disponibilidad de agua para riego. Este hecho, unido al elevado coste de la energía asociada a los sistemas de riego presurizados, hace que muchos productores demanden estrategias para mejorar la eficiencia en el uso del agua y hacer más rentable el cultivo del Ajo Morado (LOPEZ-MATA et al., 2010, LÓPEZ-URREA et al., 2003, FABEIRO CORTÉS et al., 2003, LÓPEZ-URREA et al., 2002). En este sentido Domínguez et al. (2013) desarrollaron una técnica para incrementar la eficiencia en el uso del agua mediante la combinación de las metodologías del año meteorológico típico (TMY) y del riego deficitario optimizado por etapas (ORDI), que permite distribuir de forma óptima una cantidad limitada de agua de riego, inferior a las necesidades del cultivo, maximizando el rendimiento final.

El objetivo principal de este trabajo es definir y validar estrategias de manejo del riego deficitario controlado, basadas en las metodologías TMY y ORDI (DOMíNGUEZ et al., 2012) que distribuyan de manera óptima un volumen de agua de riego limitado, durante las distintas etapas de desarrollo fenológico del Ajo Morado de Las Pedroñeras. De este modo se plantea limitar el efecto del estrés hídrico en el cultivo, maximizando el rendimiento y garantizando la calidad final del producto, para las condiciones de clima semiárido de Castilla-La Mancha.

\section{2- Materiales y métodos}

Durante la campaña 2015 se realizaron los ensayos de campo en los que se analizó el efecto de 5 tratamientos de riego sobre el rendimiento y los parámetros de calidad de la cosecha. Los tratamientos planteados fueron: uno sin déficit, y los otros cuatro con distintos volúmenes máximos de agua de riego disponible, correspondientes al 100 (T100), 90 (T90), 80 (T80) y 70\% (T70) de las necesidades netas de riego en ajo morado para las condiciones climáticas de un año meteorológico típico (TMY) intermedio (fijadas en $3400 \mathrm{~m}^{3} / \mathrm{ha}=\mathrm{T100}$ ). Este valor se obtuvo tras la calibración de los datos requeridos por el modelo MOPECO para el ajo en las condiciones de C-LM, aplicando la metodología TMY (Domínguez et al., 2013).

El uso del Modelo de OPtimización ECOnomica - MOPECO (ORTEGA et al., 2005) con las metodologías ORDI y TMY ha permitido establecer para un volumen de agua disponible, el nivel de déficit de cada fase de desarrollo, así como el calendario de riegos para cada uno de los tratamientos planteados que maximiza el rendimiento. Los objetivos de déficit y los calendarios de riegos se han ido actualizando en función de la evolución de los parámetros climáticos del año actual, haciendo nuevas optimizaciones en cada cambio de fase, permitiendo redistribuir el agua de la manera más óptima posible, respetando el volumen de agua de riego fijado para cada tratamiento (Sin déficit: sin limitación de agua, T100: $3400 \mathrm{~m}^{3} / \mathrm{ha}$, T90: $3060 \mathrm{~m}^{3} / \mathrm{ha}$, T80: $2720 \mathrm{~m}^{3} / \mathrm{ha}$ y T70: $2380 \mathrm{~m}^{3} / \mathrm{ha}$ ).

La plantación del ajo se realizó manualmente, con un marco de 0,08 metros entre plantas y 0,5 metros entre líneas de cultivo, para un área de ensayo total de $918 \mathrm{~m}^{2}$. Se realizaron cuatro repeticiones de cada tratamiento en parcelas de $2,5 \mathrm{~m} \times 18,0 \mathrm{~m}$ distribuidas al azar, salvo en los tratamientos sin déficit y el T100, donde fueron tres. El agua se aplicó mediante un sistema de riego localizado por goteo de marco cuadrado $(0,5 \times 0,5 \mathrm{~m}$ de separación entre ramales y emisores) dotado de emisores autocompensantes con un caudal nominal de 3,8 l/h. En cada sector de riego, que corresponde con un tratamiento, se instaló un caudalímetro de pulsos para controlar los volúmenes de agua aplicados. Además 
se realizaron evaluaciones de la uniformidad de aplicación de agua del sistema de riego antes de la siembra y periódicamente a lo largo del ciclo de cultivo.

En cada parcela se instalaron tensiómetros (a 20 y $40 \mathrm{~cm}$ de profundidad) y sensores de humedad volumétrica (a 10, 20, 30 y $40 \mathrm{~cm}$ ) para controlar la evolución del agua en el suelo (Figura 1). A partir de los datos ambientales registrados por la estación agroclimática completa ubicada en el campo de ensayos, de los registros de los sensores de humedad en el suelo, y de la combinación de MOPECO con las metodologías ORDI y TMY, se estableció una programación de riegos con un nivel de déficit objetivo (ETa/ETm) para cada etapa de crecimiento. Así, se fijó el calendario de riegos para cada uno de los tratamientos propuestos, sin superar en ningún caso el volumen de agua disponible prefijado.

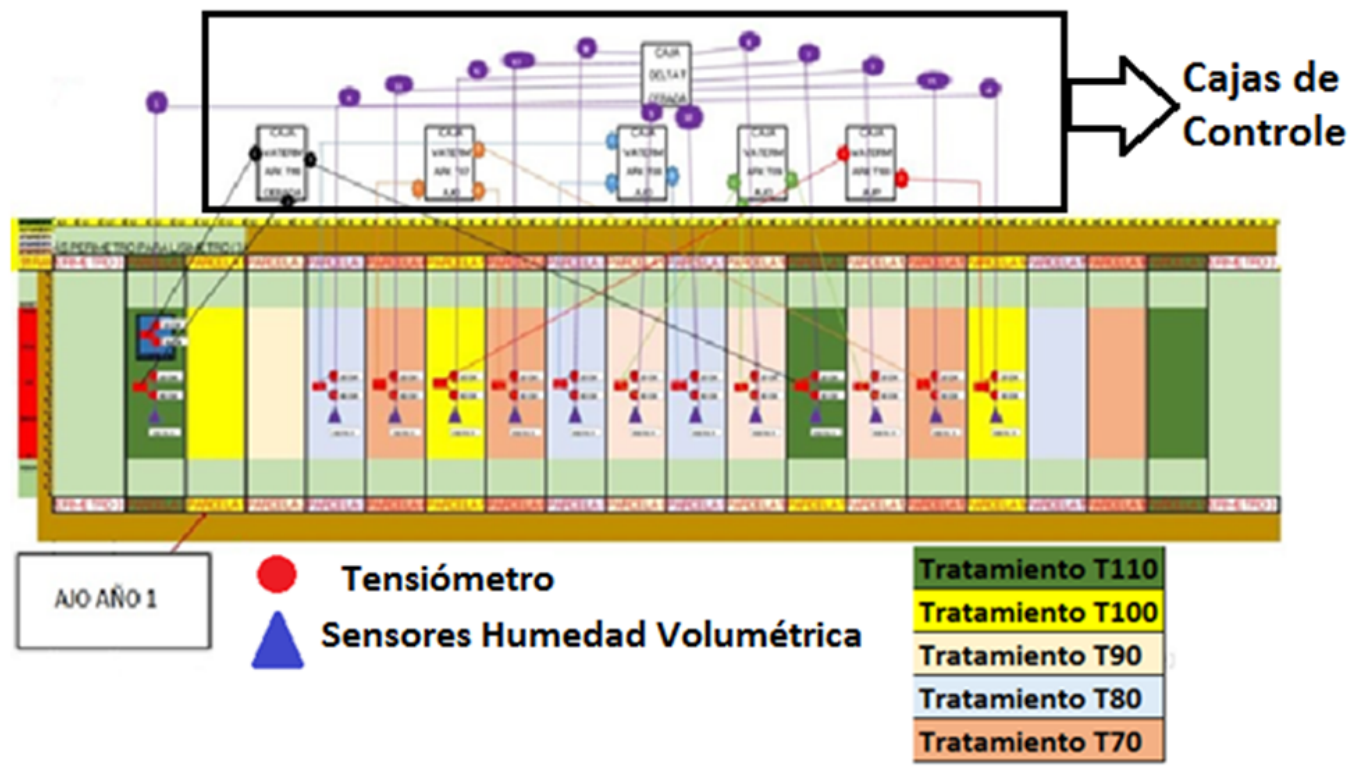

Figura 1. Esquema de la distribución de las parcelas y sensores de humedad de suelo.

Periódicamente se recogieron muestras del cultivo para analizar la evolución de parámetros fisiológicos, tales como el índice de área foliar y la biomasa. Para la determinación del índice de cobertura se tomaron fotografías RGB, mientras que para el seguimiento del nivel de estrés alcanzado por el cultivo se tomaron fotografías térmicas. Con el fin de estudiar la respuesta de la planta a los distintos déficits hídricos en fechas próximas al cambio de etapa de desarrollo fenológico, se midieron parámetros tales como la capacidad fotosintética utilizando un analizador de gases por infrarrojo, o la conductancia estomática, utilizando un porómetro.

Tras la cosecha, además del rendimiento total y su distribución por calibres, se evaluaron varios parámetros de calidad y la eficiencia del uso del agua, relacionando todo ello con los distintos tratamientos de riego aplicados y los niveles de estrés hídrico. Los parámetros analizados fueron: el color típico del Ajo Morado por medio de técnicas de colorimetría, los sólidos solubles y la textura. En condiciones de almacenamiento, se está registrando la evolución de la masa para cada uno de los tratamientos diferenciados.

\section{3- Resultados y discusión}

En el año de 2015, las optimizaciones para las tres primeras etapas de desarrollo fenológico se hicieron a partir de los datos de un TMY intermedio, y dada a la evolución climática durante el ciclo, para la última etapa se utilizaron datos de un TMY seco. Los 
valores de agua de riego neto, las relaciones entre evapotranspiración actual o real (ETa) y evapotranspiración máxima (ETm) y las obtenidas por el optimizador se presentan en la Tabla 1.

Tabla 1. Relaciones ETa/ETm optimizadas y reales para las etapas de desarrollo fenológico.

\begin{tabular}{|c|c|c|c|c|c|c|c|c|}
\hline \multirow[b]{2}{*}{2015} & \multicolumn{2}{|c|}{ Tratamiento $100 \%$} & \multicolumn{2}{|c|}{ Tratamiento $90 \%$} & \multicolumn{2}{|c|}{ Tratamiento $80 \%$} & \multicolumn{2}{|c|}{ Tratamiento $70 \%$} \\
\hline & \begin{tabular}{|c|} 
ETa/ETm \\
(Opt.)
\end{tabular} & \begin{tabular}{|c|} 
ETa/ETm \\
(Real)
\end{tabular} & $\begin{array}{c}\text { ETa/ETm } \\
\text { (Opt.) }\end{array}$ & $\begin{array}{c}\text { ETa/ETm } \\
\text { (Real) }\end{array}$ & $\begin{array}{c}\text { ETa/ETm } \\
\text { (Opt.) }\end{array}$ & $\begin{array}{c}\text { ETa/Etm } \\
\text { (Real) }\end{array}$ & $\begin{array}{c}\text { ETa/ETm } \\
\text { (Opt.) }\end{array}$ & \begin{tabular}{|c|} 
ETa/ETm \\
(Real)
\end{tabular} \\
\hline Etapa 1' & 1,00 & 1,00 & 1,00 & 1,00 & 1,00 & 1,00 & 1,00 & 1,00 \\
\hline Etapa 1" & 1,00 & 1,00 & 0,76 & 0,75 & 0,70 & 0,73 & 0,70 & 0,74 \\
\hline Etapa 2 & 1,00 & 0,91 & 1,00 & 0,91 & 1,00 & 0,91 & 0,84 & 0,80 \\
\hline Etapa 3 & 1,00 & 0,98 & 0,81 & 0,74 & 0,54 & 0,66 & 0,50 & 0,66 \\
\hline Global/Total & 1,00 & 0,95 & 0,90 & 0,84 & 0,80 & 0,81 & 0,70 & 0,76 \\
\hline \begin{tabular}{|c|} 
Riego \\
Neto(mm)
\end{tabular} & \multicolumn{2}{|c|}{253,69} & \multicolumn{2}{|c|}{188,1} & \multicolumn{2}{|c|}{179,6} & \multicolumn{2}{|c|}{159,4} \\
\hline
\end{tabular}

*Opt: Optimizado.

Al tratamiento sin déficit se le aplicó la misma cantidad de agua que al T100, por lo que no se analizaron conjuntamente. Para las programaciones de riegos establecidas, según la información proporcionada por los sensores de humedad instalados en el suelo, parece que en ningún tratamiento se alcanzaron niveles de estrés tanto en las etapas de establecimiento como al inicio del desarrollo vegetativo (Figura 2).

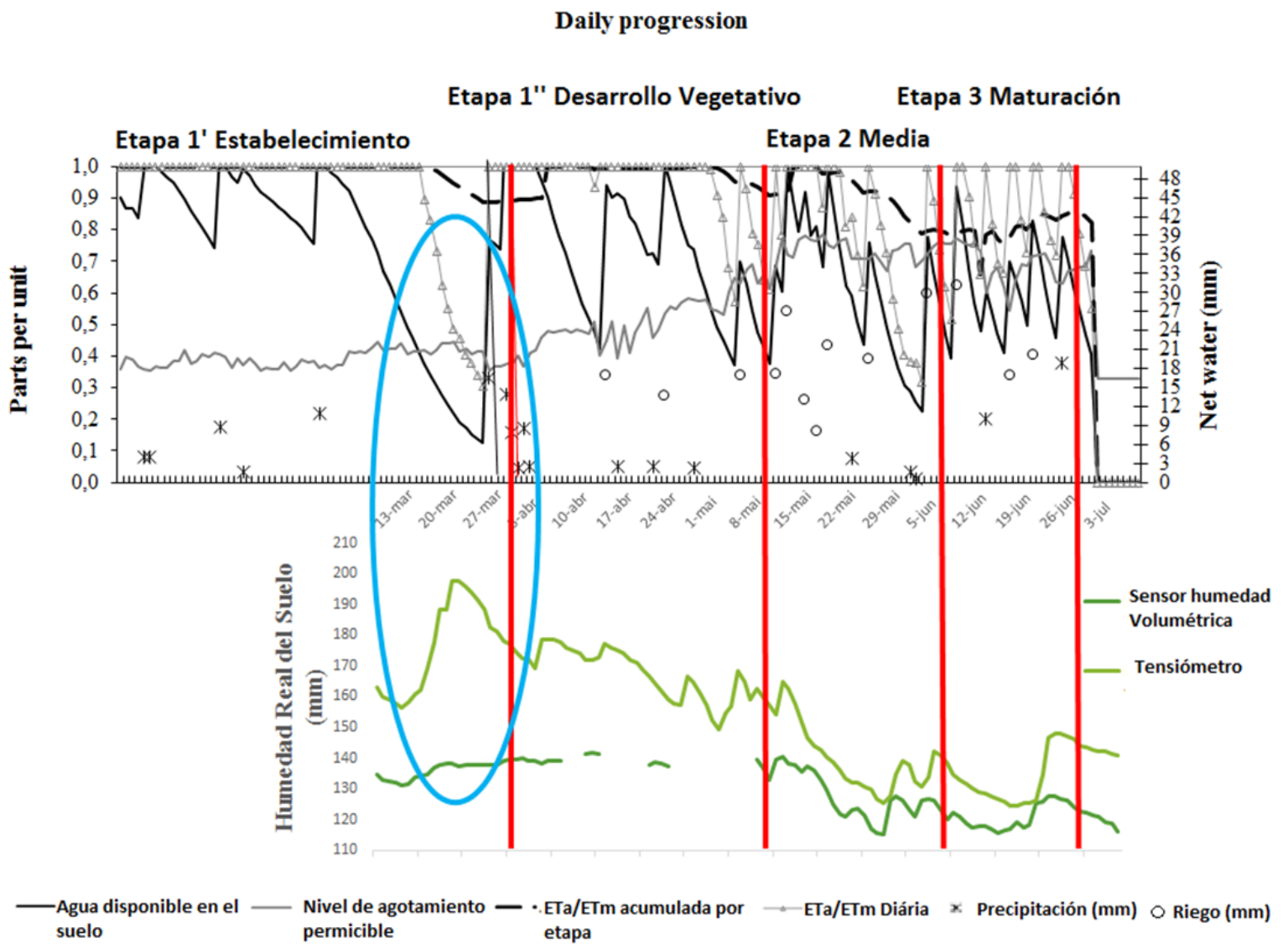

Figura 2. Comparación entre la curva de humedad del MOPECO y la curva de humedad de los sensores de suelo. 
En la parte superior de la Figura 2 se representa la evolución del agua en el suelo simulada por el modelo y en la inferior, la registrada por los sensores. En el periodo de días remarcados por el óvalo azul se presenta una diferencia significativa entre las tendencias de ambos métodos. Mientras la simulación indicaba que en las primeras etapas podría haber estrés, los sensores indicaban una elevada humedad del suelo. Esto puede ser debido a las condiciones climáticas (aparición de rocío) que podría estar aportando un agua que no ha sido considerada por el modelo para el cálculo del balance, y/o a que los valores de Kc utilizados, procedentes de la bibliografía, podrían estar sobrestimados para esta variedad.

Así, la lámina de riego necesaria para satisfacer las necesidades máximas del cultivo estimada en base a las condiciones medias, se redujo de $3400 \mathrm{~m}^{3} / \mathrm{ha}$ a $2550 \mathrm{~m}^{3} / \mathrm{ha}$. Por otro lado, la aplicación de un menor nivel de déficit hídrico al previsto durante las dos primeras etapas, debido a una menor necesidad de riego, redujo la diferencia de rendimiento entre los tratamientos (Tabla 2). En este caso, no aparecieron diferencias significativas entre tratamientos cercanos (T80 y T70), pero sí entre los más alejados (T100 y T70).

Tabla 2. Rendimientos simulados y reales para el cultivo del ajo en el año 2015.

\begin{tabular}{|c|c|c|c|c|c|c|}
\hline \multirow{2}{*}{$\begin{array}{c}\text { Agua } \\
\text { neta } \\
\left(m^{3} / h a\right)\end{array}$} & \multirow[b]{2}{*}{ TRATAMIENTO } & \multicolumn{4}{|c|}{ Rendimiento } & \multirow{2}{*}{$\begin{array}{c}\text { Diferencia } \\
\text { Rendimiento } \\
\text { Real-Simulado } \\
\text { (kg/ha) }\end{array}$} \\
\hline & & $\begin{array}{l}\text { SIMULADO } \\
(\mathrm{kg} / \mathrm{ha})\end{array}$ & $\begin{array}{l}\text { REAL } \\
\text { (kg/ha) }\end{array}$ & DV & CV & \\
\hline 3787 & T100 & 10366,2 & 10000 & 314,8 & 3,3 & $-366,2$ \\
\hline 3131 & T90 & 8724,1 & 8900 & 580,0 & 6,5 & 175,9 \\
\hline 3046 & T80 & 8410,3 & 8500 & 691,3 & 7,9 & 89,7 \\
\hline 2844 & T70 & 7892,2 & 8480 & 439,8 & 4,9 & 587,8 \\
\hline
\end{tabular}

El rendimiento en todos los tratamientos se aproxima a los valores medios de producción de la zona, que es de $8500 \mathrm{~kg} / \mathrm{ha}$ (MAGRAMA, 2016), superándolo en todos los casos excepto para el más restrictivo. La Figura 3 muestra los datos de la eficiencia del uso del agua de riego, donde se observa cómo los tratamientos más deficitarios, generan una mayor eficiencia en el uso del agua ( $\mathrm{kg}$ producidos por unidad de volumen de agua aplicada). Esto indica que el riego deficitario en el ajo puede ser apropiado para situaciones de baja disponibilidad hídrica, permitiendo aprovechar el agua que se ahorre para aplicarla a otros cultivos, o ampliar el área cultivada de ajo, creando la posibilidad de incrementar la rentabilidad total de la finca. 


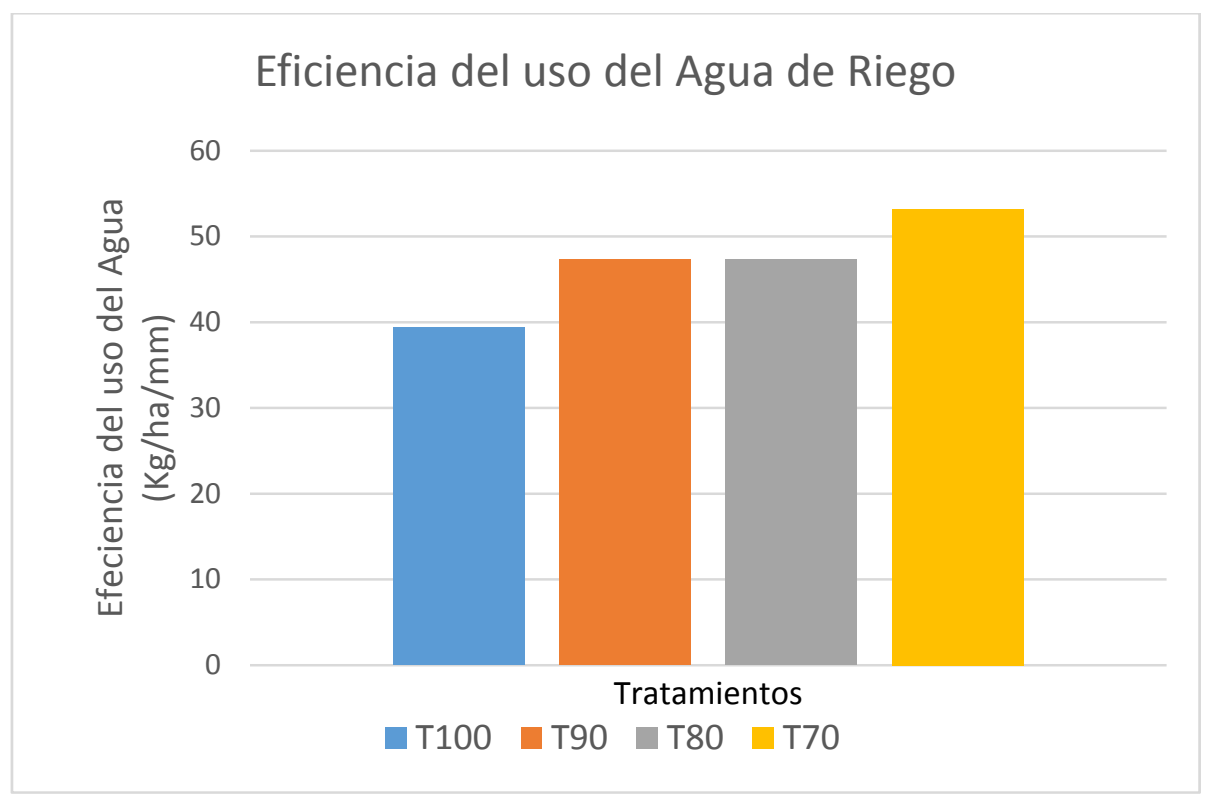

Figura 3. Eficiencia del uso del agua del riego en el cultivo de ajo e el año de 2015.

En el Ajo Morado, los parámetros cualitativos influyen directamente en el precio de venta. El color y el diámetro, son dos de los principales condicionantes de precio final. Para el año de 2015 los calibres de los ajos se encuentran en su gran mayoría en las categorías Flor (diámetro de 45-50 mm) y Súper (diámetro de 50-55 mm), que son las más demandadas comercialmente (Figura 4).

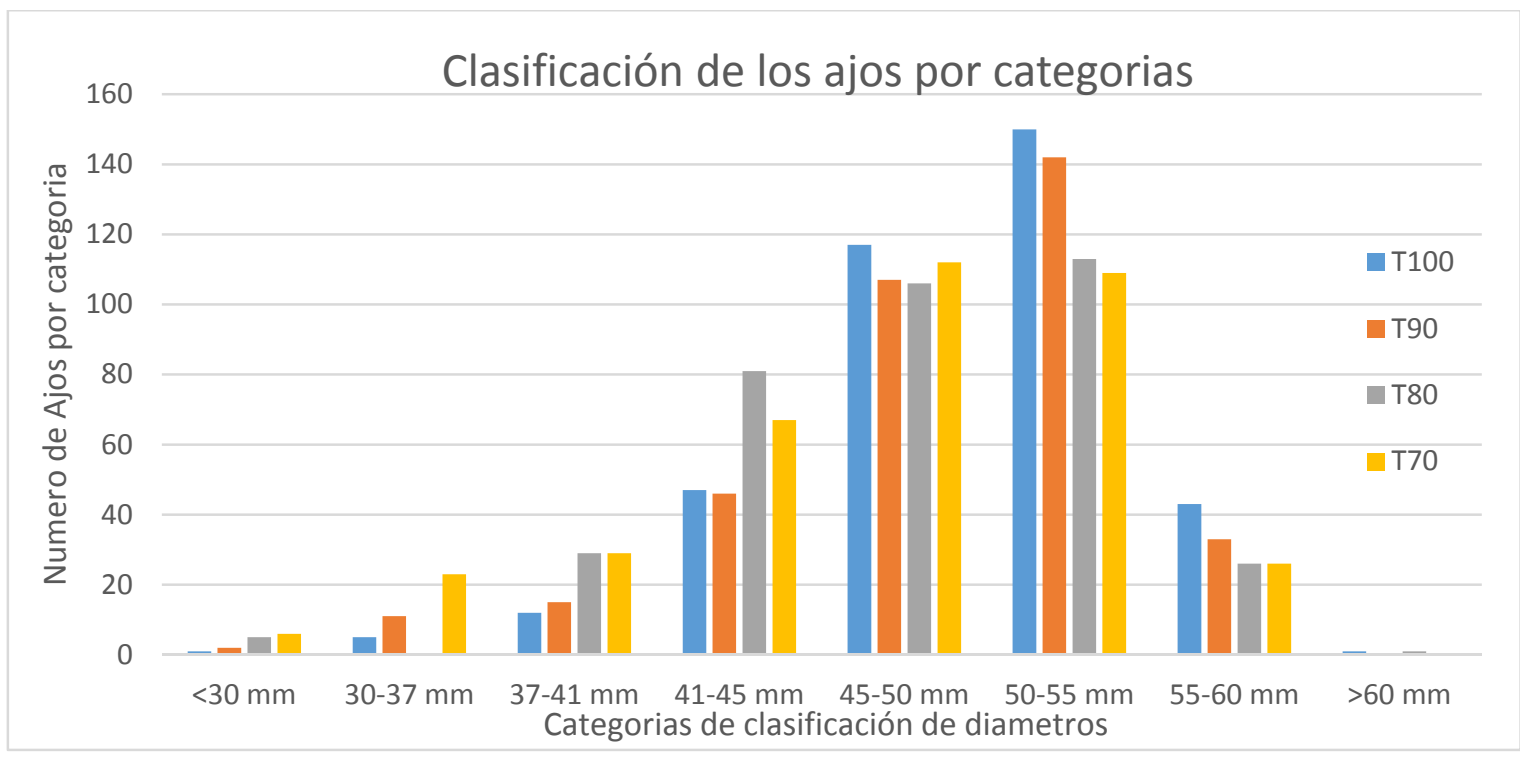

Figura 4. Clasificación de las cabezas de Ajo Morado según la categoría.

En la Tabla 3 se presenta el valor medio de los paramentos físicos (coordenadas cromáticas $\left(L^{*}, a^{*}, b^{*}\right)$, atributos cromáticos $\left(C^{*}, h^{*}\right)$ ) relacionados con el color. Para el análisis de colorimetría se compararon los distintos tratamientos y una muestra de un patrón de color considerado óptimo para la comercialización. No se detectaron diferencias significativas entre tratamientos, ni entre éstos y la muestra tomada como patrón. Todo ello indica que para los niveles de estrés hídrico generados, no hay una disminución de la calidad referida al color.

Tabla 3. Valores medios de los parámetros físicos de colorimetría para el Ajo Morado. 


\begin{tabular}{|c|c|c|c|c|c|}
\hline TRATAMIENTO & $\mathbf{L}^{*}$ & $\mathbf{a}^{*}$ & $\mathbf{b}^{*}$ & $\mathbf{C}^{*}$ & $\mathbf{h}^{*}$ \\
\hline T100 & $66,83^{\mathrm{a}}$ & $12,04^{\mathrm{a}}$ & $11,84^{\mathrm{a}}$ & $17,40^{\mathrm{a}}$ & $45,64^{\mathrm{a}}$ \\
\hline T90 & $63,11^{\mathrm{a}}$ & $13,74^{\mathrm{a}}$ & $11,43^{\mathrm{a}}$ & $18,30^{\mathrm{a}}$ & $40,32^{\mathrm{b}}$ \\
\hline T80 & $63,75^{\mathrm{a}}$ & $13,36^{\mathrm{a}}$ & $12,19^{\mathrm{a}}$ & $18,56^{\mathrm{a}}$ & $44,05^{\mathrm{a}}$ \\
\hline T70 & $64,46^{\mathrm{a}}$ & $13,38^{\mathrm{a}}$ & $12,88^{\mathrm{a}}$ & $18,96^{\mathrm{a}}$ & $44,72^{\mathrm{a}}$ \\
\hline Referencia & $63,11^{\mathrm{a}}$ & $13,74^{\mathrm{a}}$ & 11,43 & $18,30^{\mathrm{a}}$ & $40,32^{\mathrm{b}}$ \\
\hline
\end{tabular}
(Test de Duncan).

En cuanto a la textura, el tratamiento T100 presentó la media de resistencia a penetración más elevada, aunque no se encontraron diferencias significativas en relación a los demás tratamientos y la muestra de referencia facilitada por la empresa colaboradora. El valor medio para los sólidos solubles en todos los tratamientos fue de $39^{\circ} \mathrm{BRIX}$, superior al presentado como referencia por otros autores (PARDO et al., 2003) para otras variedades de ajo morado, con aproximadamente $28^{\circ} \mathrm{BRIX}$. El análisis de la variación de la masa durante el almacenaje se ha realizado durante un año a partir de la fecha de almacenamiento (21 de septiembre de 2015), por lo que todavía no hay datos concluyentes respecto a la influencia de los tratamientos.

Con los valores obtenidos en los muestreos, realizados periódicamente durante todo el ciclo de desarrollo del ajo, se calcularon los índices de área foliar (LAl) y biomasa. La evolución de los valores de LAl para las distintas fechas, fue similar en todos los tratamientos (Figura 5); con respecto a biomasa, el T100 presentó una ligera diferencia al final del ciclo (Figura 5).
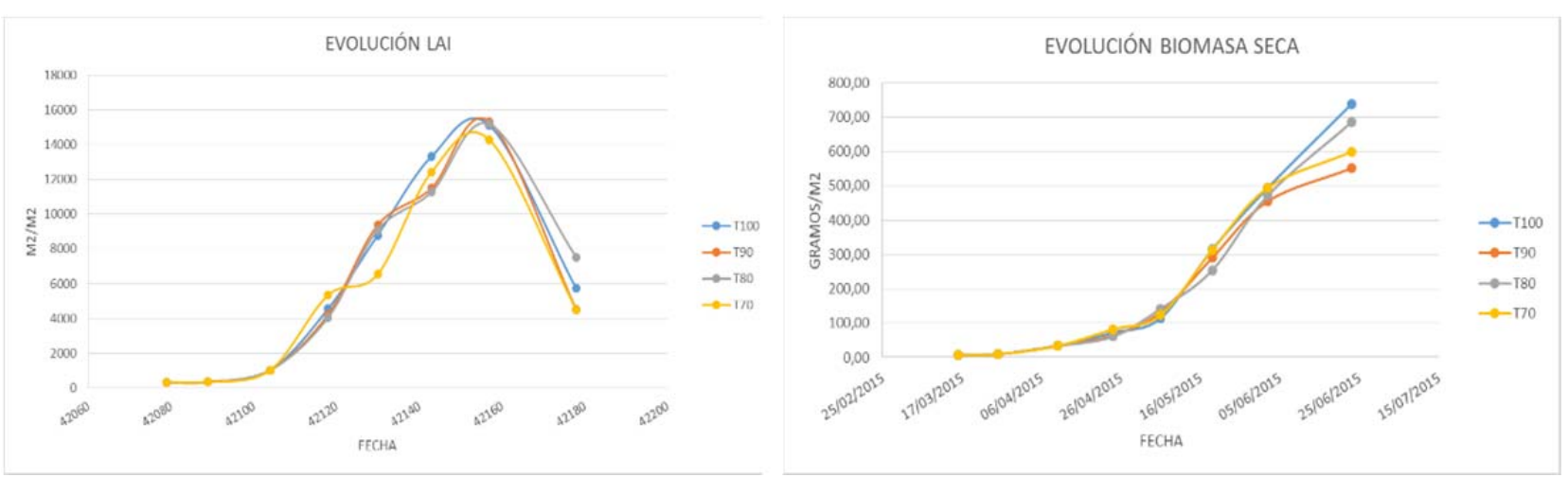

Figura 5. Evolución del área foliar (LAI) y de la biomasa seca del ajo para los diferentes niveles de agua de riego en el año 2015.

Al final de la segunda etapa de desarrollo, para disminuir el nivel de humedad del suelo, se redujo el volumen de riego, forzando la planta a consumir la reserva de agua disponible. Esto, generó en el cultivo los primeros síntomas de estrés, que fueron registrados por las lecturas del porómetro. Los valores más altos de resistencia estomática se dieron en todos los tratamientos el día 8 de mayo (Figura 6). 


\section{EVOLUCIÓN RESISTENCIA ESTOMÁTICA AJO 2015}

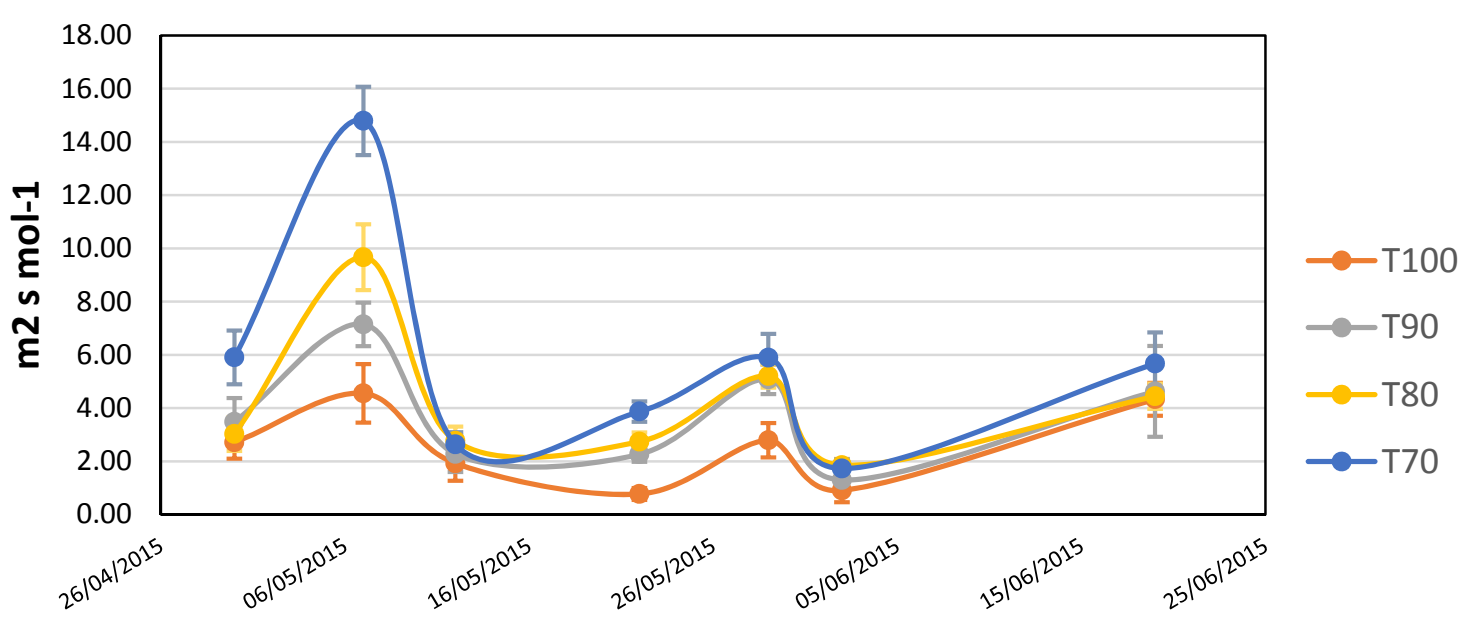

Fecha

Figura 6. Evolución de la resistencia estomática en el cultivo del ajo en el año de 2015.

El T70 fue el tratamiento que presentó el valor de resistencia estomática más elevado durante todo el ciclo, aunque la dificultad que ello conlleva para realizar los intercambios gaseosos y consecuentemente la fotosíntesis, no derivó en pérdidas significativas en la asimilación de carbono y el crecimiento vegetativo, tal como fue descrito anteriormente.

\section{4- Conclusiones y recomendaciones}

La metodología empleada logró un nivel adecuado, tanto en lo referido a los rendimientos como a la calidad de la cosecha en todos los tratamientos. Esto fue debido en parte a que las necesidades del ajo en la zona son inferiores a las inicialmente previstas, especialmente durante la fase de establecimiento e inicio del desarrollo vegetativo. Es interesante realizar un seguimiento de la evolución en los valores de rocío en la zona, que permitan ajustar los kc para la variedad de Ajo Morado de la Pedroñeras.

\section{5- Bibliografía}

Domínguez, A., de Juan, J.A., Tarjuelo, J.M., Martínez, R.S., Martínez-Romero, A., 2012.Determination of optimal regulated deficit irrigation strategies for maize in a semi-arid environment. Agric.Water Manage. 110, 67-77

Domínguez, A., Martínez-Romero, A., Leite, K.N., Tarjuelo, J.M., de Juan, J.A., López-Urrea, R., 2013. Combination of typical meteorological year with regulated deficit irrigation to improve the profitability of garlic in central Spain. Agric.Water Manage. 130, 154-167.

Fabeiro Cortés, C.,Martín de Santa Olalla, F., López Urrea, R., 2003. Production of garlic (Allium sativum L.) under controlled deficit irrigation in a semi-arid climate. Agric.Water Manage. 59, 155-167. 
FAO, 2016. Food and Agricultural Commodities Production. Food and Agriculture Organization of the United Nations, www.fao.org (Consulta 31.03.16)

López-Mata, E., Tarjuelo,J.M., de Juan, J.A., Ballesteros, R.,Domínguez, A., 2010. Effect of irrigation uniformity on the profitability of crops. Agric. Water Manage. 98, 190-198.

López-Urrea, R., López, H., López, P., Fabeiro, C., Martín de Santa Olalla, F.J., 2002. Ensayo de riego deficitario controlado en ajo morado. In: Anuario Técnico ITAP 2001. Instituto Técnico Agronómico Provincial,www.itap.es/ITAPPublicaciones/4Publicaciones/Publicaciones.asp (Consulta 31 de Marzo 2016).

López-Urrea, R., López, H., López, P., Fabeiro, C., Martín de Santa Olalla, F.J., 2003. Ensayo de riego deficitario controlado en ajo morado. In: Anuario Técnico ITAP 2002. Instituto Técnico Agronómico Provincial, www.itap.es/ITAPPublicaciones/4Publicaciones/Publicaciones.asp (Consulta 31 de Marzo 2016).

MAGRAMA, 2016. Anuario de estadística $2014 . \quad$ Avance: http://www.magrama.gob.es/estadistica/pags/anuario/2014-avance/AE 2014 13.pdf; Ministerio de Agricultura, Alimentación y Medio Ambiente, Consulta 31 de marzo 2016.

Ortega, J.F., de Juan, J.A., Tarjuelo, J.M., 2005. Improving water management: the Irrigation Advisory Service of Castilla-La Mancha (Spain). Agric.Water Manage. 77 (1), 37-58.

Pardo, J.E., González, M.E., Escribano, J., Granell, J.D., Alvarruiz, A. 2003. Evaluación de la calidad de variedades seleccionadas de ajo I.Parámetros físico-químicos. Alimentaria. Diciembre 03, 121-125. 